\title{
TRATAMENTO CIRÚRGICO DA DOENÇA PILONIDAL SACROCOCCÍGEA: ESTUDO COMPARATIVO ENTRE AS TÉCNICAS DE MARSUPIALIZAÇÃO E DE INCISÃO E CURETAGEM
}

\author{
SURGICAL TREATMENT OF SACROCOCCIGEAL PILONIDAL DISEASE: \\ A COMPARATIVE STUDY BETWEEN MARSUPIALIZATION AND INCISION \\ PLUS CURETTAGE TECHNIQUES
}

\author{
Sérgio Norberto Zárate Arauz ${ }^{1}$ \\ Nagamassa Yamaguchi, TCBC-SP2 \\ Fábio Schmidt Goffi, ECBC-SP ${ }^{3}$ \\ Arnaldo Zanoto ${ }^{4}$
}

\begin{abstract}
RESUMO: Objetivo: Comparar duas técnicas cirúrgicas empregadas, no tratamento da doença pilonidal, a de marsupialização e da incisão e curetagem, analisando seus resultados imediatos, e tardios. Método: A casuística de 52 pacientes que apresentaram doença pilonidal sacrococcígea em suas diferentes fases, divididos em dois grupos. Grupo A, com 23 doentes submetidos à técnica de marsupialização, e grupo B, formado por 29 doentes operados pela técnica de incisão e curetagem. Todos tiveram seguimento pós-operatório de um a três anos, no período de 1981 a 1999 pelo Serviço de Gastroenterologia Cirúrgica do Hospital do Servidor Público Estadual "Francisco Morato de Oliveira" de São Paulo. A média de idade do grupo A foi de 25,39 \pm 8,92 anos e a do grupo B foi de 25,52 \pm 9,98 anos. A distribuição por sexo foi de 28 mulheres e 24 homens. Segundo a raça, foram 42 brancos e 10 negros. Resultados: A média de tempo de duração de cirurgia, para o grupo A, submetido à marsupialização, foi de 38,91 \pm 19,77 minutos, e para o grupo B, doentes submetidos à incisão e curetagem, foi de 47,34 $\pm 14,34$ minutos. A média do tempo de internação para o grupo A foi de 3,26 dias e para o grupo B 1,28 dia. Quanto ao uso de antibiótico, em ambos grupos não foi observada diferença estatística (p>0,05). O tempo médio de cicatrização da ferida operatória nos doentes submetidos à técnica de marsupialização foi de 29,30 $\pm 13,24$ dias e para os doentes submetidos a incisão e curetagem foi de 40,76 $\pm 26,56$ dias. A dor pós-operatória incidiu com $72,4 \%$ dos doentes do grupo B, e de $17,4 \%$ do grupo A, observando-se significância estatística $(\mathrm{p}=0,0001)$. $\mathrm{O}$ grupo de doentes submetidos à marsupialização não apresentou sangramento, enquanto no grupo da incisão e curetagem 20,7\% dos enfermos tiveram sangramento $(\mathrm{p}=0,2829)$. A incidência de infecção pós-operatória não diferiu em ambos grupos (4,4\% e 3,5\%, respectivamente). O grupo B apresentou maiores índices de recidivas $(17,6 \%)$ em relação ao grupo A $(4,3 \%)$ com diferença estatisticamente significante $(\mathrm{p}=0,3364)$. Conclusões: Os procedimentos cirúrgicos empregados são de fácil execução, com períodos per-operatórios aceitáveis. A cicatrização parece ser mais eficiente nos doentes operados pela técnica de marsupialização. A técnica de incisão e curetagem apresentou maior número de complicações: dor e sangramento com maior frequiência que na técnica de marsupialização. A técnica de incisão e curetagem apresentou maior recidiva que a técnica de marsupialização.
\end{abstract}

Descritores: Doença pilonidal sacrococcígea; Técnica cirúrgica; Marsupialização; Curetagem.

\section{INTRODUÇÃO}

Embora a doença pilonidal sacrococcígea seja conhecida há muito tempo e diversas variedades terapêuti- cas tenham sido propostas, não há consenso sobre a melhor estratégia para abordá-la. Do ponto de vista cirúrgico, diversos procedimentos foram sugeridos: a zetaplastia $^{1-3}$, a fenolização do trajeto fistuloso ${ }^{4}$, a excisão com

1. Mestre em Medicina - Bolsista da CAPES - Fundação de Coordenação de Aperfeiçoamento de Pessoal de Nível Superior. PEC/PG

2. Doutor em Medicina - Professor Doutor da FMUSP

3. Professor Emérito da FMUSP - Presidente da CPG-IAMSPE

4. Assessor Estatístico do Programa de Pós-graduação do IAMSPE

Recebido em 06/11/2001

Aceito para publicação em 11/06/2002

Trabalho realizado no Serviço de Gastroenterologia Cirúrgica do Hospital do Servidor Público Estadual "Francisco Morato de Oliveira", São Paulo. 
fechamento primário ${ }^{5}$ e a excisão com fechamento por segunda intenção $0^{6}$.

Cirurgiões pretendem alcançar a cura da doença por meio de operações de pequena envergadura e pouca complexidade, que permitam rápida integração dos doentes às suas atividades habituais ${ }^{7}$. Neste contexto, duas técnicas foram freqüentemente utilizadas: a de marsupialização ${ }^{8,9}$, e a de incisão e curetagem ${ }^{10-16}$.

O intuito deste trabalho foi realizar estudo comparativo retrospectivo entre as técnicas de marsupialização e da incisão e curetagem, objetivando aferir dados para melhor subsidiar a opção terapêutica.

\section{MÉTODO}

A casuística constou de 52 doentes operados no período de 1981 a 1999 no Serviço de Gastroenterologia Cirúrgica do Hospital do Servidor Público Estadual "Francisco Morato de Oliveira" de São Paulo, que apresentaram a doença pilonidal sacrococcígea em suas diferentes fases. A idade variou de 13 a 58 anos, com média de 25,45 $\pm 9,45$ anos. Quanto ao sexo eram 28 mulheres e 24 homens. Segundo a distribuição por raça, 42 brancos e dez negros. $\mathrm{O}$ seguimento pós-operatório variou de um a três anos.

Os 52 doentes foram divididos em dois grupos. Grupo $\mathrm{A}$, com 23 doentes submetidos à técnica de marsupialização, e o grupo B, formado por 29 doentes operados pela técnica de incisão e curetagem.

Todos os doentes de ambos os grupos foram submetidos à seguinte preparação pré-operatória:

1. Pré-operatório: avaliação clínica geral e constatação da evidência da doença pilonidal sacrococcígea; tricotomia da região a ser operada, antes do paciente ser levado ao centro cirúrgico.

2. Anestesia: foram usadas anestesia local e raquidiana.

3. Posição operatória dos doentes: os doentes foram colocados em decúbito ventral com a mesa cirúrgica em semiflexão, fazendo ligeira proeminência da região sacrococcígea (posição de Navalha Sevilhana ou de Depage). Foram coladas tiras de fita adesiva (esparadrapo) nas nádegas para fazer tração lateral e melhorar a exposição da região sacrococcígea. Em seguida feita anti-sepsia na região com tintura de Polivinil Pirrolidona Iodo $1 \%$ (Povidine ${ }^{\circledR} 1 \%$ Johnson, Brasil), foram colocados os panos estéreis para delimitar a área cirúrgica.

\section{TéCniCa de InCisão e Curetagem OU DE ZIMMERMAN}

O primeiro passo foi a verificação dos orifícios do cisto, sinus ou fístula com a introdução de estilete ou tentacânula e demarcação da área a ser excisada em forma de elipse. Logo se fez ressecção da pele que recobre a cavidade do cisto, permanecendo exposta a parede interna ou leito do mesmo.

A seguir realizou-se curetagem do tecido de granulação e dos pêlos que estavam no interior do cisto. Posteriormente foram procurados trajetos secundários para se fazer o mesmo procedimento que o trajeto primário já exposto anteriormente.

Realizou-se hemostasia rigorosa com eletrocautério, tamponamento com gaze furacinada e curativo compressivo, dando por terminado o procedimento cirúrgico da incisão e curetagem.

\section{Técnica de Marsupialização ou de Buie}

Além de se realizar todos os primeiros passos da técnica de incisão e curetagem descrita anteriormente, iniciou-se a sutura das bordas da pele ao tecido do leito curetado do cisto. Usou-se fio categute cromado $2 / 0$ ou $3 /$ 0 com pontos interrompidos, ficando, dessa maneira, o cisto completamente marsupializado para iniciar o período de cicatrização e epitelização por metaplasia. A ferida foi coberta com curativo furacinado e compressivo.

As variáveis qualitativas foram representadas por frequiência absoluta (n) e relativa (\%). As variáveis quantitativas foram representadas por média, desvio padrão (DP), mediana, valores mínimos e máximos, usando Quiquadrado $\left(\chi^{2}\right)$ com correção de Yates, teste exato de Fisher, teste $\mathrm{t}$ de Student, cálculos de percentuais, tabela de contingência 2 x 2 e gráficos de barra, segundo o caso.

Adotou-se o nível de significância de $5 \%(\mathrm{a}=0,05)$. Os níveis descritivos (p) inferiores a esse valor, considerados significantes, foram representados por *.

Foram analisadas as seguintes variáveis: tempo de anestesia, duração da cirurgia, tempo de internação, uso de antibióticos, tempo de cicatrização, complicações como dor, sangramento e infecção e, por último, recidiva da doença.

\section{RESULTADOS}

O tempo médio de duração da cirurgia, para o grupo submetido à marsupialização, foi de 38,91 $\pm 19,77$ minutos e para os doentes submetidos a incisão e curetagem foi de 47,34 \pm 14,34 minutos; não foi encontrada diferença estatística significante $(\mathrm{p}=0,0810)$.

Com respeito ao tempo de internação, para o grupo A a média foi de 3,26 dias e para o grupo B, 1,28 dia. Observou-se que os doentes do grupo B tiveram menor tempo de internação. Obteve-se diferença estatística $(\mathrm{p}=0,5120)$.

O uso de antibiótico, em ambos grupos, não apresentou diferença (Tabela 1).

\section{Tabela 1}

Doentes que usaram antibiótico e que foram submetidos à técnica de marsupialização e de incisão e curetagem

\begin{tabular}{l|c|c|c}
\hline Antibiótico & Marsupialização & \multicolumn{1}{c}{$\begin{array}{c}\text { Incisão } e \\
\text { curetagem }\end{array}$} & Total \\
\hline Sim & $1(4,4 \%)$ & $6(20,7 \%)$ & 7 \\
Não & $22(95,6 \%)$ & $23(79,3 \%)$ & 45 \\
\hline Total & 23 & 29 & 52 \\
\hline
\end{tabular}

Fisher $p=0,11705$

$N S=$ não significante. 
O tempo de cicatrização, para os doentes submetidos à técnica de marsupialização (grupo A) foi de 29,30 $\pm 13,24$ dias e para os pacientes submetidos à incisão e curetagem (grupo B) foi de 40,76 $\pm 26,56$ dias. Embora no grupo A tenha sido menor o tempo de cicatrização que no grupo B, não houve diferença estatisticamente significante $(\mathrm{p}=0,0650)$.

No contexto das complicações pós-operatórias, a dor apresentou-se mais freqüentemente no grupo B de doentes com incisão e curetagem. Observou-se significância estatística $(\mathrm{p}=0,0001 *)$ (Tabela 2).

A freqüência do sangramento pós-operatório é mostrada na Tabela 3. O grupo A (técnica de marsupialização) não apresentou sangramento, enquanto no grupo B (técnica de incisão e curetagem) houve ocorrência de $20,7 \%(\mathrm{p}=0,02829 *)$.

Quanto à ocorrência da infecção pós-operatória não houve diferença estatística entre ambos grupos $(\mathrm{p}=1,00000)$ (Tabela 4).
A análise das recidivas da doença é apresentada na Tabela 5, mostrando maior índice no grupo B, com significância estatística $(\mathrm{p}=0,03364)$.

\section{DISCUSSÃO}

O uso de antibióticos está indicado apenas nos quadros agudos. Alguns autores, entretanto, advogam seu uso contínuo com o intuito de manter a área livre de bactérias e ajudar a cicatrização ${ }^{17-20}$.

Em nosso estudo o tempo de cicatrização em doentes operados pela técnica de marsupialização foi, em média, de 29,30 $\pm 13,24$ dias e pela de incisão e curetagem, de 40,76 $\pm 26,56$ dias.

A literatura consigna que a cicatrização pela técnica de fechamento primário dura, em média, 15 a 27 dias; já no método de excisão, com fechamento por segunda intenção, chega a 56 dias, com a inconveniência de que o manuseio da ferida nos curativos é muito doloroso para o doente $21-23$.

Tabela 2

Presença do sintoma dor pós-operatória nos doentes submetidos aos dois diferentes procedimentos cirúrgicos

\begin{tabular}{l|rr|rrr}
\hline Dor pós-operatória & Marsupialização & Incisão e curetagem & Total \\
\hline Presente & 4 & $(17,4 \%)$ & 21 & $(72,4 \%)$ & 25 \\
Ausente & 19 & $(82,6 \%)$ & 8 & $(27,6 \%)$ & 27 \\
\hline Total & 23 & 29 & 52 \\
\hline
\end{tabular}

Fisher $p=0,0001 *$

$*$ = significante

Tabela 3

Sangramento pós-operatório apresentado nos grupo de técnica de marsupialização e de incisão e curetagem

\begin{tabular}{l|rr|r|r}
\hline Sangramento pós-operatório & Marsupialização & Incisão e curetagem & Total \\
\hline Presente & $0 \quad(0,0 \%)$ & $6 \quad(20,7 \%)$ & 6 \\
Ausente & $23(100,0 \%)$ & $23 \quad(79,3 \%)$ & 46 \\
\hline Total & 23 & 29 & 52 \\
\hline
\end{tabular}

Fisher $p=0,02829$

$*$ = significante

Tabela 4

Infecção pós-operatória apresentada no grupo A (marsupialização) e grupo B (incisão e curetagem)

\begin{tabular}{l|rr|rrr}
\hline Infecção pós-operatória & Marsupialização & Incisão e curetagem & Total \\
\hline Presente & 1 & $(4,4 \%)$ & 1 & $(3,5 \%)$ & 2 \\
Ausente & 22 & $(95,6 \%)$ & $28 \quad(96,5 \%)$ & 50 \\
\hline Total & 23 & 29 & 52 \\
\hline
\end{tabular}

Fisher $p=1,00000$

$N S=$ não significante. 
Tabela 5

Recidivas pós-operatórias nos doentes operados pela técnica de marsupialização (grupo A) e a técnica de incisão e curetagem (grupo B)

\begin{tabular}{|c|c|c|c|}
\hline Recidivas & Marsupialização & Incisão e curetagem & Total \\
\hline Presente & $1 \quad(4,3 \%)$ & $8 \quad(27,6 \%)$ & 9 \\
\hline Ausente & $22(95,7 \%)$ & $21 \quad(72,4 \%)$ & 43 \\
\hline Total & 23 & 29 & 52 \\
\hline
\end{tabular}

Fisher $p=0,03364$

* = significante

No método de incisão e curetagem, o tempo médio de cicatrização alcança de 27 a 45 dias ${ }^{13-16}$. No entanto, com a técnica de marsupialização a cicatrização varia de 20 a 30 $\operatorname{dias}^{7,19,24}$.

A cicatrização mais rápida da ferida após a marsupialização é atingida com as seguintes condutas: 1- completa excisão e retirada do tecido enfermo; 2- mobilização adjacente da ferida, somente o necessário, para se aproximar, sem tensão; 3- hemostasia completa; 4- boa obliteração dos espaços mortos. Tais recomendações foram feitas por Corman ${ }^{9}$, e praticadas nos doentes de nossa casuística operados por esta técnica.

A cicatrização é obtida através do processo de obliteração da superfície seguida de epitelização da mesma. Durante esse período as pontes devem ser divididas para que se obtenha boa epitelização ${ }^{25,26}$, tal como foi feito em nossos pacientes.

Na técnica aberta, com cicatrização por segunda intenção, procura-se manter a área cirúrgica limpa e livre de detritos e/ou pêlos ${ }^{13,27}$.

A técnica de marsupialização foi descrita pela primeira vez por Buie ${ }^{28}$, durante a Segunda Grande Guerra. Nessa ocasião o autor define a doença como "Doença do Jipe". Seguidos pelos impulsos dos bons resultados da técnica de marsupialização, outros autores a adotaram 29 - 32 . Recentemente, vários cirurgiões $7,18,24,33$ alcançaram bons resultados e as recidivas variaram de $1,2 \%$ a $5 \%$, com tempo de acompanhamento dos pacientes por períodos de três a seis anos.

Paralelamente aos avanços da técnica de marsupialização, a técnica de incisão e curetagem foi desenvolvida e realizada inicialmente com sucesso por Jacob ${ }^{34}$,seguido por outros ${ }^{35-38}$.

No âmbito nacional, Silva ${ }^{13}$, acérrimo defensor da técnica, apresenta bons resultados, com recidivas ao redor do $1,25 \%-4 \%$. Outros autores ${ }^{39,40}$ assinalam maiores índices de recidivas, que variaram de $4,4 \%$ a $19,5 \%$.

Autores nacionais que usam a técnica de incisão e curetagem apresentam recidivas pós-operatórias ao redor $3,5 \%$, no acompanhamento de três a oitos anos ${ }^{14-16}$. O fato é discordante quando anotamos $27,3 \%$ de recidivas no seguimento de um a três anos.
Dor e desconforto pós-operatório se apresentam em torno de $28 \%$ dos doentes nas técnicas abertas em geral, sendo aconselhável, para prevenir estas complicações, que as feridas não sejam muito largas. Caso isto ocorra prefere-se que o doente permaneça hospitalizado ${ }^{21}$.

Embora tenham sido obedecidas estas recomendações, nossa casuística apresentou índice maior de dor pósoperatória em $72,4 \%$ dos doentes operados pela técnica de incisão e curetagem. Obtivemos índices menores para a técnica de marsupialização $(17,4 \%)$.

A técnica de marsupialização não acarreta, em termos gerais, desconforto pós-operatório. É indolor e dispensa qualquer cuidado especial. Outros autores têm relatado períodos prolongados de cicatrização e, em determinadas ocasiões, o fio usado para fazer a marsupialização atua de corpo estranho para formar granulomas nas feridas ${ }^{4,9,19}$. Tal complicação não ocorreu em nosso estudo.

O sangramento pós-operatório surge, na literatura, ao redor de $3 \%$ 20,25. Nossos dados referem a ocorrência de sangramento em $20,7 \%$ dos pacientes utilizando a técnica de incisão e curetagem, e zero, na técnica de marsupialização.

Outras complicações observadas na literatura foram a separação da ferida cirúrgica em $6 \%$, infecção ou abscesso em 30\%, hematomas em 3\%, infecção após o fechamento primário em $5,4 \%$ a $35 \%$. Descreveu-se, também, que o hematoma é a causa mais freqüente de retardo na cicatrização, o que ocorre de $1 \%$ a $43 \%{ }^{20,25}$. Este fato não foi por nós confirmado.

Recomenda-se que após a cirurgia se mantenha boa higiene, com depilação ao redor da área cirúrgica, já que isso previne a migração dos pêlos à ferida. Esse fato, que faz retardar a cicatrização, é confirmado nos estudos de Surrell ${ }^{41}$ e Dorton ${ }^{42}$.

Finalmente $\mathrm{Close}^{43}$ sugeriu que não importa o método cirúrgico executado, mas sim saber quando e como usá-lo adequadamente. A maioria das falhas encontra-se na má avaliação pré-operatória e nos cuidados pós-operatórios insatisfatórios dos pacientes. A sugestão foi praticada meticulosamente em nossos doentes. 


\begin{abstract}
Objective: Results of two surgical techniques for treatment of pilonidal disease, marsupialization and incision and curettage were compared. Method: Fifty-two patients with sacrococcygeal pilonidal disease were divided into two groups: Group A consisted of 23 patients treated with marsupialization technique, and group $B$ was composed of 29 patients submitted to incision and curettage. Postoperative follow-up varied between one and three years, from 1981 to 1999. Mean age for group A was $25.39 \pm 8.92$ years and for group B $25.52 \pm 9.98$ years. There were 28 females and 24 males. Results: The average duration of the surgery for group $A$ was 38.91 \pm 19.77 minutes and for group B $47.34 \pm 14.34$ minutes. The mean in-ward duration time was 3,26 days for group A and 1.28 day for group B. Regarding antibiotic use, no statistical difference was observed between groups ( $p>0.05)$. Average wound healing time associated with marsupialization was $29.30 \pm 13.24$ days, and for incision and curettage $40.76 \pm 26.56$ days. Postoperative pain occurred in $72.4 \%$ of the patients in group B and $17,4 \%$ of group A $(p=0,0001)$. Wound bleeding was absent in patients of group A but occurred in $29.7 \%$ of patients of group $B(p=0,02829)$. The incidence of postoperative infection did not differ between the two groups (4.4\% and $3.5 \%$ respectively). Group B presented a higher reccurring rate $(27.6 \%)$ compared to group $A$ $(4.3 \%)(p=0,03364)$. Conclusions: Healing appears to be more efficient in patients undergoing marsupialization technique. Incision and curettage technique presents greater number of complications, with pain and bleeding occuring more often than with the marsupialization technique. The incision and curettage technique has a higher recurring rate than the marsupialization technique.
\end{abstract}

Key Words: Sacrococcigeal pilonidal disease; Surgical technique; Marsupialization; Curettage.

\title{
REFERENNCIAS
}

1. Monro RS, McDermott FT. The elimination of causal factors in pilonidal sinus treated by Z-platy. Br J Surg 1965; 52, 177-298.

2. Hodgson WJB, Greenstein RJ. A comparative study between Z-plasty and incision and drainage or excision with marsupialization for pilonidal sinuses. Surg Gynecol Obstet 1981.; 153, 842-44.

3. Mansoory A, Dickson D. Z-platy for treatment of disease of the pilonidal sinus. Surg Gynecol Obstet 1982; 155, 409-11.

4. Duchateau J, Demol J, Bostoen H, Allegaert W. Pilonidal sinus excision - marsupialization - phenolization? Acta Chir Belg 1985; 85:325-28.

5. Lord PH, Millar DM. Pilonidal sinus: A simple treatment. Br J Surg 1965: 52:298-300

6. Marks J, Harding KG, Hughes LE, Ribeiro CD. Pilonidal sinus excision - healing by open granulation. $\mathrm{Br} \mathrm{J}$ Surg 1985;72:637-640.

7. Vaula JL, Badaro JA, Nacusse E, Cadierno A. Enfermedad pilonidal sacrococcígea. Prensa Med Argent 1986; 73:489-91.

8. Buie LA. Jeep disease (pilonidal disease of mechanized warfare). South Med J 1944; 37:103-9.

9. Corman ML. Classic article in colonic and rectal surgery. Dis Colon Rectum 1982; 25:384-90.

10. Zimmerman K. Surgical treatment of pilonidal disease. J Intern Coll Surg 1955; 24:104-6.

11. Zimmerman K. Pilonidal disease. Lancet 1958;1:46-9.

12. Zimmerman K. Pilonidal disease. Dis Colon Rectum 1970;13:330-2. 1970.

13. Silva JH. Cisto pilonidal sacrococcígeo. Contribuição ao tratamento cirúrgico pela técnica da incisão e curetagem. 1972. 86f. Tese (Doutorado em Medicina). Faculdade de Medicina da Universidade de São Paulo.
14. Nahas SC, Sobrado CW, Araújo SEA, Imperiale AR, HarbGram A, Pinotti HW. Resultados do tratamento de cisto pilonidal não complicado. Rev Hosp Clin Fac Med S Paulo 1997;52:287-90.

15. Silva JH. Cisto pilonidal. In: Silva JH. Manual de Coloproctologia. São Paulo: Associação Paulista de Medicina, 2000a. p195-210.

16. Silva JH. Pilonidal cysts. Cause and treatment. Dis Colon Retum 2000b;43:1146-56.

17. Fraiha A, Habr-Gama A, Goffi FS. Resultados o tratamento cirúrgico do cisto piloso sacro pelos métodos aberto e fechado. Rev Assoc med bras 1969;15:89-92.

18. Cavanagh CR, Schnug E, Girvin GW, McGonigle DJ. Definitive marsupialization of the acute pilonidal abscess. Am Surg 1970;36:650-1.

19. Figueiredo EV, Melo, PVNF, Rangel MF. Tratamento da doença pilonidal pela técnica de marsupialização. Ars Cvrandi gastroenterol 1985;4:37-40.

20. Lundhus E, Gottrup P. Outcome at three to five years of primary closure of perianal and pilonidal abscess. Eur J Surg 1993;159:555-8.

21. Kooistra HP. Pilonidal sinuses. Review of the literature and report of three hundred fifty case. Am J Surg v1942;40:3-17.

22. Solla JA, Rothenberger DA. Chronic pilonidal disease. An assessment of 150 cases. Dis Colon Rectum 1990;33:758-61.

23. Allen-Mersh TG. Pilonidal sinus. Finding the right track for treatment. Br J Surg 1990;72:123-32.

24. Meban S, Hunter E. Outpatient treatment of pilonidal disease. Can Med Assoc J 1982;126:941.

25. Abramson DJ. A simple marsupialization technic for treatment of pilonidal sinus: long-term follow-up. Ann Surg 1960;151:261-67.

26. Goodall $\mathrm{P}$. The aetiology and treatment of pilonidal sinus. A review of 163 patients. Br J Surg 1996;49:212-18. 
27. Davage ON. The original of sacrococcygeal pilonidal sinuses. Based on an analysis of our hundred sixty - three cases. Am J Path 1954;30:1191-204.

28. Buie LA, Curtiss RK. Pilonidal disease. Surg Clin North Am 1952;32:1247-59.

29. Coffey RJ. Marsupialization of pilonidal cyst. US Nav Med Bull 1944;42:1326-9.

30. Paterson P, Ames RH. Pilonidal sinuses and cysts. Am J Surg 1944;65:384-90.

31. Brockbank MJ, Floyd JR. Pilonidal cyst. Am J Surg 1945;68:77-9.

32. Freund SJ, Redding MD. Marsupialization. Am J Surg 1948;76:286-8.

33. Van Ess LJ. Surgical management of pilonidal disease. Am J Proctol 1965;16:389-93.

34. Jacobs RG. Observation on pilonidal cyst. US Nav Med Bull 1943;41:1296-8.

35. Fergunson C. Pilonidal cyst: their hospitalization. Milit Surg 1946;98:307-9.

36. Marks MM. Pilonidal sinus: Treatment by eventration. South Med J 1947;40:844-8.

37. Marks MM. Repair of painful sacral scars. South Med J 1949;42:319-23.

38. Marks MM. Recurrent pilonidal disease a misnomer: its management and treatment. J Int Coll Surg 1957;27:226-8.

39. Edwards MH. Pilonidal sinus: a 5-years appraisal of the Millar-Lord treatment. Br J Surg 1977;64:867-8.

40. Bissett IP, Isbister WH. The management of patient with pilonidal disease - a comparative study. Aust N Z J Surg 1987;57:939-942.
41. Surrell JA. Pilonidal disease. Surg Clin North Am 1994; 74:1309-15.

42. Dorton HE. Conservative treatment of pilonidal disease a foreign body granulomatous process. Am Surg 1970;36:349-51.

43. Close AS. Pilonidal cysts: an analysis of surgical failures. Ann Surg 1955;141:523-6.

Endereço para correspondência:

Dr. Fábio Schmidt Goffi

Av. Nove de Julho, 4.861/70 andar

01407-200 — São Paulo-SP 